\title{
Reverberation mapping of AGNs through continuum polarization
}

\author{
P. A. Rojas Lobos, R. W. Goosmann, J. M. Hameury, and F. Marin
}

Université de Strasbourg, CNRS, Observatoire Astronomique de Strasbourg, UMR 7550, 67000 Strasbourg, France
e-mail: jean-marie.hameury@astro.unistra.fr

Received 25 February 2020 / Accepted 8 April 2020

\begin{abstract}
Context. The size and geometry of the broad-line region (BLR) in active galactic nuclei (AGNs) are among the main ingredients in determining the mass of the accreting black hole. Size and geometry can be constrained by determining the delay between the optical continuum and the flux reprocessed by the BLR, in particular, through the emission lines.

Aims. We propose here that the delay between polarized and unpolarized light can also be used in much the same way to constrain the size of the BLR; we verify that meaningful results can be expected from observations using this technique.

Methods. We used our code STOKES to simulate polarized radiative transfer. We determined the response of the environment of the central source (BLR, dust torus, and polar wind) to randomly generated fluctuations in the central source. We then calculated the cross correlation between the simulated polarized flux and the total flux to estimate the time delay that would be provided by observations using the same method.

Results. The BLR is the main contributor to the delay between the polarized flux and the total flux. This delay is independent of the observation wavelength.

Conclusions. This validates the use of polarized radiation in the optical/UV band to estimate the geometrical properties of the BLR in type I AGNs, in which the viewing angle is close to pole-on and the BLR is not obscured by the dust torus.
\end{abstract}

Key words. galaxies: active - galaxies: Seyfert - polarization - radiative transfer - scattering

\section{Introduction}

Since the discovery of broad emission lines in the polarized spectrum of NGC 1068 (Miller \& Antonucci 1983; Antonucci \& Miller 1985) and 3C 234 (Antonucci 1984), a radio-loud AGN, our current view of an active galactic nucleus (AGN) has been that of an accreting black hole surrounded by its accretion disk, and, lying close to the equatorial plane, in increasing distance order, the broad line region (BLR) and a dust torus (Antonucci 1993). At much larger distances of about some hundreds of parsecs, the narrow line region (NLR) is found and can be spatially resolved. Outflows in the form of a polar wind (PW) and/or a jet may also form and connect the central engine to the NLR. Differences in the viewing angle of these systems account for most of the differences in their observational appearance: when the AGN is seen close to edge-on, the BLR is hidden by the dust torus, and broad lines are normally not observed; the AGN is of type 2. On the other hand, when the inclination is such that the BLR is directly visible, the AGN is of type 1 .

Except for the NLR and the jet at large distances, none of these regions could be spatially resolved until very recently, and our knowledge about their structure was therefore indirect. Recent interferometric observations of the quasar 3C 273 (Gravity Collaboration 2018a) and of Sgr A* (Gravity Collaboration 2018b) have indeed been able to provide spatial information on the innermost parts of AGNs: of the BLR in the case of 3C 273, and of regions at a few Schwartzschild radii in the case of Sgr $\mathrm{A}^{*}$. A few other sources have been observed using interferometry techniques: first, NGC 1068 with the MiD-Infrared Interferometric Instrument MIDI on the Very large Telescope Interferometer (VLTI; Jaffe et al. 2004;
Raban et al. 2009) or with ALMA (García-Burillo et al. 2016), and later, a few others with Gravity (Gravity Collaboration 2020), which have partially resolved the hot dust regions. These observations, however, are often limited to visibility measurements for nearby sources. The Event Horizon Telescope has provided images of the immediate vicinity of the central black hole of M87 (Event Horizon Telescope Collaboration 2019), but these cannot constrain the more distant regions, such as the external parts of the accretion disk or the BLR. Despite these spectacular progresses, adding new observational tools is therefore most important; polarimetry has proven in recent years that it can be very efficient for this purpose (see, e.g., the detection of broad lines in the polarized spectrum of NGC 1068 by Antonucci \& Miller 1985, which is strong evidence that type 1 and type 2 AGNs form a unified class) because it traces photons that have scattered in the environment of the central source.

Determining the mass of the central supermassive black hole in AGNs is a key ingredient for understanding the structure, formation, and evolution of these objects, and more generally, of galaxies themselves. Several techniques can be used, among which reverberation mapping is popular because of its simplicity. In this technique, the motion of the BLR clouds is assumed to be virialized; their velocity can be estimated from the line velocities, and their distance to the central black hole is determined by the delay between variations of the continuum flux and variations of the line intensity (see, e.g., Blandford \& McKee 1982; Peterson 1993; Zu et al. 2011). Delays of a few days are usually observed (Lira et al. 2018), corresponding to BLR sizes a few light-days, but longer delays have been found in a number of cases, such as CTS 252 (190 days in the quasar rest frame, Lira et al. 2018), and CTS C30.10 (about 560 days in the 


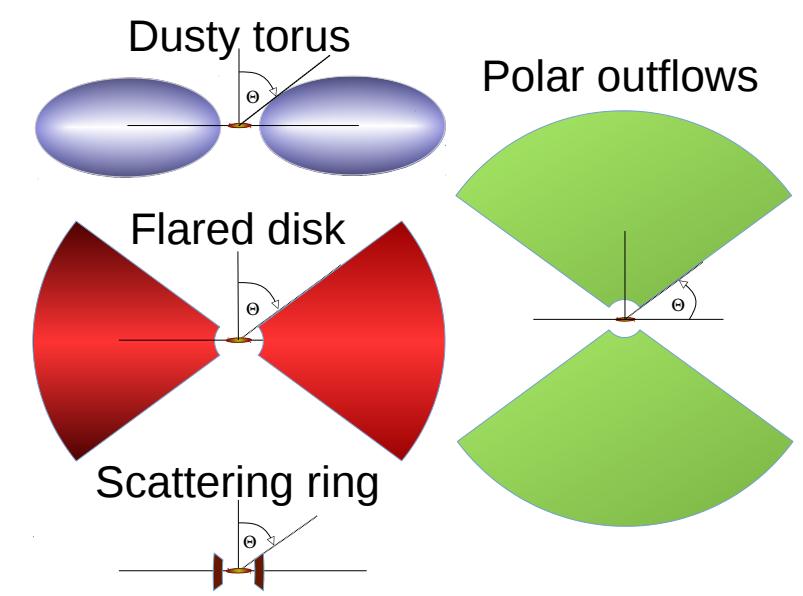

Fig. 1. Geometry. Top left: classical torus geometry (blue) with elliptical cross section. Middle left: extended flared-disk geometry (red) with the wedge-shaped cross section. Bottom left: scattering ring (brown) representing the BLR. Right: polar outflows (green). Figures from Rojas Lobos et al. (2018).

rest frame, Czerny et al. 2019). The mass of the central object directly follows from Newton's law.

In this paper, we consider another possible way to constrain the size of the BLR: we use the delay between variations of the polarized and the continuum flux. This method has been proposed in Shoji et al. (2005) and was later used by Gaskell et al. (2012), who found that the polarized flux in the $B$ band in NGC 4151 follows the total flux in the $B$ band with a delay of $8 \pm 3$ days. However, polarization of the optical light in AGNs arises from scattering of the continuum that is emitted by the central source on the environment of the black hole, which includes the BLR, but also other circumnuclear regions such as the polar wind, and the dust torus. In the case of the BLR and the polar wind, scattering is usually considered to be due to electrons, whereas in the case of the torus, scattering is due to dust. In some models, dust scattering plays an important role in the BLR (see, e.g., Czerny \& Hryniewicz 2011; Marin \& Goosmann 2013; Czerny et al. 2017), but these are not considered here. We therefore need to verify that these outer regions do not significantly affect the conclusion that might be drawn from the determination of this lag. The measured time-lag also needs to be precisely related to the properties of the BLR.

In a previous paper (Rojas Lobos et al. 2018, hereafter Paper I), we calculated the average time delay of polarized photons that underwent one or several scatterings compared to photons that propagated from the central source without any interaction. This mean delay, however, is impossible to determine directly from observations. The reason is that it includes the contribution of scattering by distant regions that would be very difficult to observe because the propagation time might be longer than the observation period over which luminosity variations are observed, and also because the ability of detecting time lags depends on the coherence properties of the central source signal. The very notion of "average" delay is also poorly defined; it can refer to the standard mean or to the median, for instance. These two quantities may differ very significantly, as we show here.

We here compare observations of the predicted time-lag for scattered photons. We simulate observations of an AGN seen at low inclination, assuming that the central source varies randomly. In Sect. 2 we convolve the source luminosity with the transfer function that we obtain using polarized transfer simulations form our STOKES code, which was initially
Table 1. Parameters of the scattering regions.

\begin{tabular}{lcccc}
\hline \hline Component & Torus & Flared disk & BLR & Polar wind \\
\hline$r_{\text {in }}(\mathrm{pc})$ & 0.061 & 0.061 & 0.0067 & 0.0067 \\
$r_{\text {out }}(\mathrm{pc})$ & 15.061 & 15.061 & 0.0577 & 30.0067 \\
$\theta\left({ }^{\circ}\right)$ & 60 & 60 & 60 & 30 \\
$\tau_{\text {opt }}$ & 150 & 150 & 1 & 0.3 \\
Composition & Dust & Dust & Electrons & Electrons \\
\hline
\end{tabular}

developed by Goosmann \& Gaskell (2007) and further upgraded by Marin et al. (2012, 2015), Marin (2018) in order to determine the predicted polarized and unpolarized fluxes. We do not include the contribution of the host galaxy that dilutes the light from the central source (Marin 2018), but because the starlight contribution is not expected to vary on short timescales, this probably does not affect our results. We note, however, that correcting for the starlight contribution is crucial to determine the slope of the BLR radius-luminosity relation (Bentz et al. 2013). We then calculate the cross correlation of the polarized and total flux, and we present our results in Sect. 3. We show that the cross correlation of the polarized and total flux is mainly sensitive to scattering in the BLR, and when the optical depth of the BLR is not too large, provides a measurement of its outer radius. We stress that our aim is not to reproduce the eight-day delay observed by Gaskell et al. (2012) in NGC 4151, but rather to test the method and determine the dependence of the predicted time-lag on the geometry and on the parameters of the system.

\section{Model}

Figure 1 shows the geometry we used here, which is the same as in Rojas Lobos et al. (2018): a central point source surrounded by the BLR, modeled as a scattering ring with inner and outer ring radius $r_{\mathrm{s}, \text { in }}$ and $r_{\mathrm{s} \text {,out }}$, respectively. This ring has a conical cross section with a half-opening angle $\theta_{\mathrm{s}}$. This equatorial scattering region is required to account for the polarization properties of Seyfert 2 galaxies (Smith et al. 2004); its optical depth must not be too low if this ring is to play any role at all, and it cannot be too high either. Smith et al. (2004) considered a ring with a Thomson optical depth of one. The dusty absorber is assumed either to have the same shape with parameters $r_{\mathrm{t}, \mathrm{in}}, r_{\mathrm{t}, \text { out }}$, and $\theta_{\mathrm{t}}$, or to have a doughnut shape. The dimensions we considered here are the same as in Paper I, and are given in Table 1. The dust torus and flared disk can be either homogeneous or clumpy; in the latter case, the filling factor is $25 \%$ and the cloud radius is $0.2 \mathrm{pc}$ in the torus case, and $0.6 \mathrm{pc}$ in the flared disk case. Outflows in AGNs are optically thin, with $\log N_{\mathrm{H}}$ usually in the range 20-22 for warm absorbers and 22-24 for ultrafast or non-ultrafast outflows (Tombesi et al. 2013). We considered here a polar wind with total radial optical depths of 0.3 , clearly at the upper end of the allowed range, in order to maximize its effects.

In the following, the inclination $\Phi$ of the AGN is taken to be $30^{\circ}$ ( $\Phi=0$ for a face-on AGN), that is, the viewing angle corresponds to a type 1 AGN.

\subsection{Source term}

The response of the environment of the central source can be written as

$L(\lambda, t)=\int_{0}^{\infty} \Psi(\lambda, \tau) S(t-\tau, \lambda) \mathrm{d} \tau$ 
where $L$ is the observed luminosity, $S$ is the time-dependent luminosity of the central source, and $\Psi$ is the transfer function, that is, the response of the system to an impulsive source luminosity, $S(t)=\delta(t)$. All quantities depend on the observation wavelength $\lambda$. We considered three possible values of $\lambda$, corresponding to the visible (5500 $\AA$ ), blue (4500 $\AA$ ), and ultraviolet $(2500 \AA)$ domains. $\Psi$ was calculated using our STOKES code, which was modified in order to calculate the path difference between scattered photons and photons that directly reach the observer without interactions.

The time variability of AGNs is often described by a damped random walk (Kelly et al. 2009; Zu et al. 2013), in which a correcting term is added to a random walk model to push the deviations back to their mean value for timescales longer than a characteristic timescale of some months to years, depending on the black hole mass (MacLeod et al. 2010). For timescales shorter than this characteristic timescale, both processes give similar light curves, and the power spectrum density (PSD) is a power law with index -2 . For very short timescales, Kepler observations of AGNs (Aranzana et al. 2018) have shown that the PSD at high frequencies $\left(10^{-6}-10^{-4} \mathrm{~Hz}\right.$, corresponding to timescales of a few hours to some days) is still a power law, but the index varies from source to source, usually by about -2 to -3 . More general methods exist for simulating a light curve, such as the one proposed by Timmer \& Koenig (1995) for generating a signal with a power-law PSD with a given index, or the continuous-time autoregressive moving average (CARMA) model (Kelly et al. 2014), in which the PSD can be expressed as a sum of Lorentzian functions, and is therefore extremely flexible and able to model a broad range of PSDs.

Because we are interested in timescales of some days to weeks, and in order to avoid introducing additional free parameters, we generated the source light curve according to a random walk scheme,

$t_{\mathrm{i}}=i \Delta t$

$S\left(t_{\mathrm{i}}\right)=S\left(t_{i-1}\right)+2 \beta\left(r_{\mathrm{i}}-1 / 2\right)$,

where $r_{\mathrm{i}}$ is a random number uniformly distributed in the range $[0-1], \beta$ is a constant that determines the variability of the source light curve, taken here to be $\beta=0.07$, and $\Delta t$ is the time interval between two discrete source points, taken here to be 0.60 days. We verified numerically that the PSD of the light curve generated according to this process was indeed a power law with index -2 . The value of $\beta$ determines the rms variation of the source observed on a timescale $\Delta t$; it varies from source to source and depends on the observation wavelength as well as on the degree of dilution of the AGN light by the host galaxy. As we show below (see Fig. 7), a $\beta$ of 0.07 results in variations by factors of two on timescales of a few years, in agreement with what is observed in NGC 4151 (Gaskell et al. 2012), for example. Our method therefore completely omits the red part of the PSD, as well as the high-frequency component. We discuss in Sect. 3 the effect of the statistical model we used to describe the source term on the delays that can be obtained, and we show that our results are reliable and do not depend significantly on the assumptions made on the time variability of the source.

\subsection{Transfer function}

\subsubsection{Equatorial ring}

Figure 2 shows the transfer function that we obtained considering the scattering by an equatorial ring alone. The total flux is

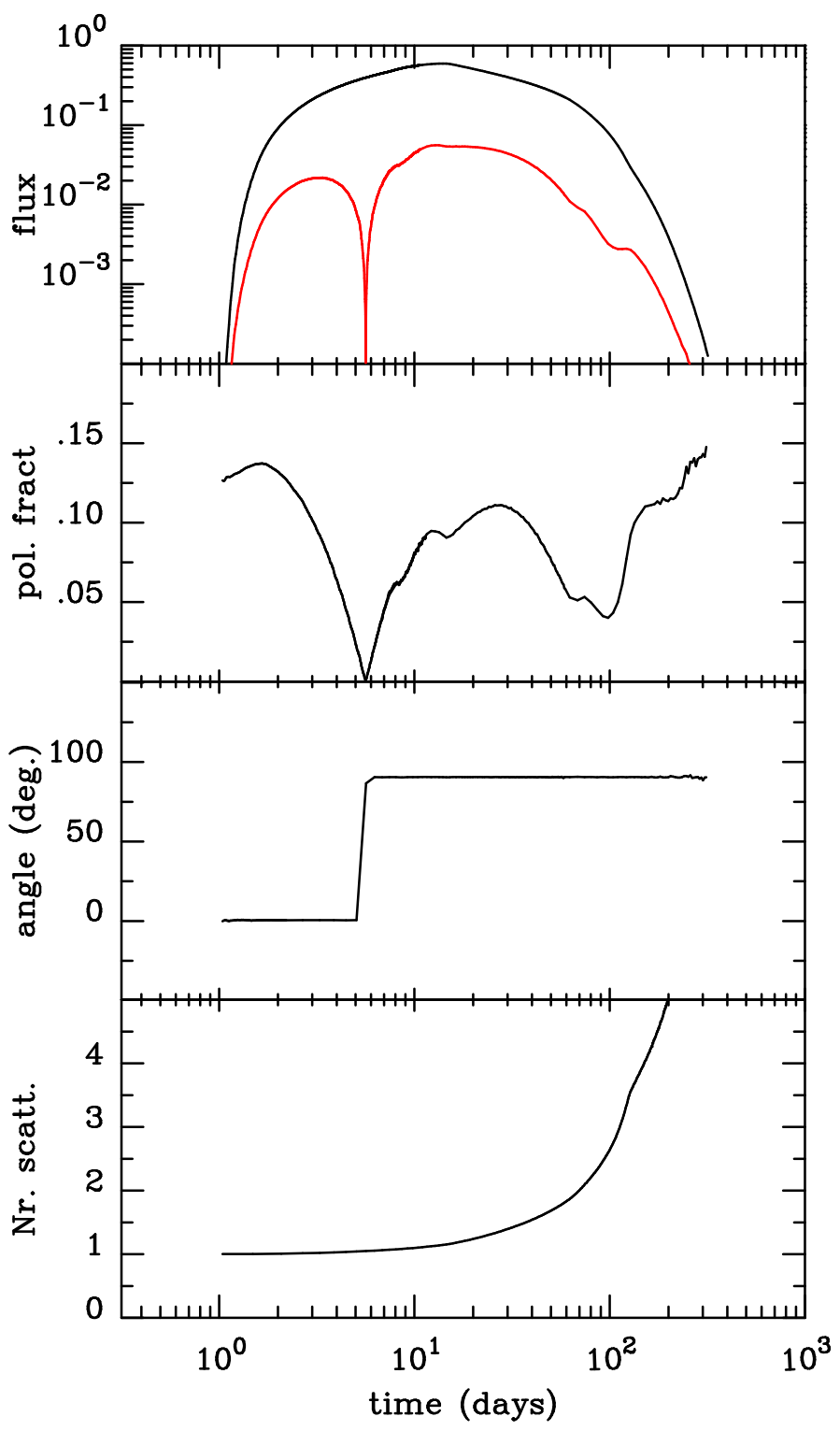

Fig. 2. Transfer function obtained from STOKES for the equatorial ring alone. From top to bottom: total (red) and polarized (black) flux, polarization fraction, polarization angle, and number of electron scattering as a function of time lag. The viewing angle is $30^{\circ}$.

the sum of a delta function for a zero time-lag (not shown in the figure because the $x$-axis is logarithmic) that corresponds to photons reaching the observer without scattering, and of the scattered component than spans a broad range of time lags. There is a minimum value because the ring does not extend to the central black hole. This is given by

$\tau_{\min }=[1-\cos (\Phi-\theta)] r_{\text {in }} / c$.

For the parameters considered here, the minimum time-lag is 1.1 days, slightly shorter than the first data point in Fig. 2. The polarized flux (red line) clearly shows two maxima at 3 days and 12 days. The first maximum corresponds to the case where scattering occurs in the part of the ring that is closest to the observer, and the second maximum is due to scattering on the opposite side of the ring. This also accounts for the change in the polarization angle between the two maxima: the angle is 0 for short delays, corresponding to scattering by the fraction of the BLR that is closest to the observer, and is $90^{\circ}$ for 
longer delays, corresponding to a situation where the full inner BLR contributes. The position of the first maximum decreases with increasing viewing angles, and disappears when the viewing angle reaches $55^{\circ}$, while the position of the second and main maximum does not depend significantly on the viewing angle. The polarized fraction is quite significant, about $10 \%$ to $15 \%$, as expected when the scattering angle is close to $\pi / 2$ (Chandrasekhar 1960), and is slightly higher for the first than for the second maximum. For time delays of up to a few months, the average number of scatterings of polarized photons is very close to unity because the optical depth of the ring is moderate: we considered an optical depth of one here. Long time delays correspond to multiple scatterings and have a low probability, hence the decrease in both the total and polarized flux. The maxima of the transfer function are relatively broad.

The time lag corresponding to an "average" scattering photon should correspond to a maximum of $\tau \Psi(\tau)$ that is proportional to the probability that the time lag is in the range $[\tau, \tau(1+\epsilon)]$, where $\epsilon$ is some fixed quantity. In the case shown in Fig. 2, this maximum occurs for a time lag of 33 days, which is longer by a factor 2 than the position of the maximum of $\Psi$. The maximum of $\tau \Psi(\tau)$ corresponds quite well to the median time lag $\tau_{\mathrm{m}}$, which is defined such that an equal number of photons suffer a lag shorter or longer than $\tau$, and that is equal to 31 days. It is shorter than the mean time lag, defined as

$\langle\tau\rangle=\frac{\int_{0}^{\infty} \tau \Psi(\tau) \mathrm{d} \tau}{\int_{0}^{\infty} \Psi(\tau) \mathrm{d} \tau}$,

and that is equal to 43 days in the case considered here. The difference between $\tau_{\mathrm{m}}$ and $\langle\tau\rangle$ is due to the long tail of $\Psi$.

This average scattering photon interacts inside the scattering ring at a distance of typically $r_{\text {out }} / 2$ to $r_{\text {out }} / 3$ because the optical depth is unity; this distance is $0.02-0.03 \mathrm{pc}$, and the corresponding propagation time is 24 to 36 days, accounting for a geometrical factor that is slightly smaller than unity for the inclination considered here. A secondary maximum, corresponding to the first peak seen in Fig. 2, is smaller by a factor 15 than the main peak of $\tau \Psi(\tau)$, and therefore plays no major role.

\subsubsection{Dust torus}

In the case of a dusty torus, time delays are much longer. Figure 3 shows the transfer function in the case where the torus is uniform. The two maxima correspond, as for the equatorial ring, to the inner parts of the torus that are closest to (farthest from) the observer. The torus is optically thick, and the average scattered photon is expected to have interacted with the circumnuclear medium in regions where the optical depth is about unity. By the geometrical shape of the torus, these regions are mostly located close to the inner edge of the torus. More precisely, in the case of a uniform medium, the radial optical depth at a point located at a distance $r$ from the central black hole is $\left(r-r_{\text {in }}\right) /\left(r_{\text {out }}-r_{\text {in }}\right) \tau_{\text {tot }}$, where $\tau_{\text {tot }}$ is the total optical depth of the torus. An optical depth of two-thirds is reached at a distance $r=r_{\text {in }}+r_{\text {out }} / \tau_{\text {tot }}$ because $r_{\text {out }}$ is much larger than $r_{\text {in }}$ For the parameters considered here, this occurs at a distance of $0.12 \mathrm{pc}$, and hence the time lag of an average scattered photon is about 140 days, times a geometrical factor slightly smaller than unity. This compares well with the peak of $\tau \Psi(\tau)$ that occurs at $\tau=92$ days. The median time lag is $\tau_{\mathrm{m}}=98 \mathrm{~d}$, in good agreement with the position of this maximum, and is again far smaller than the mean lag $\langle\tau\rangle=127 \mathrm{~d}$.

The situation is quite similar for the flared disk case.

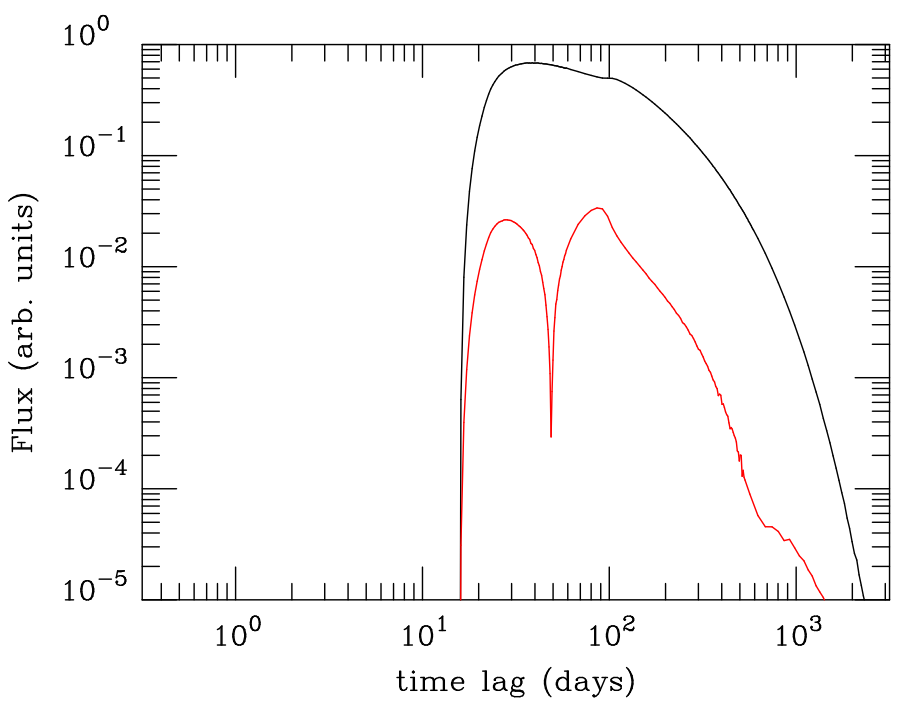

Fig. 3. Transfer function obtained from STOKES for a uniform dust torus alone. The total (black) and polarized (red) fluxes are shown.

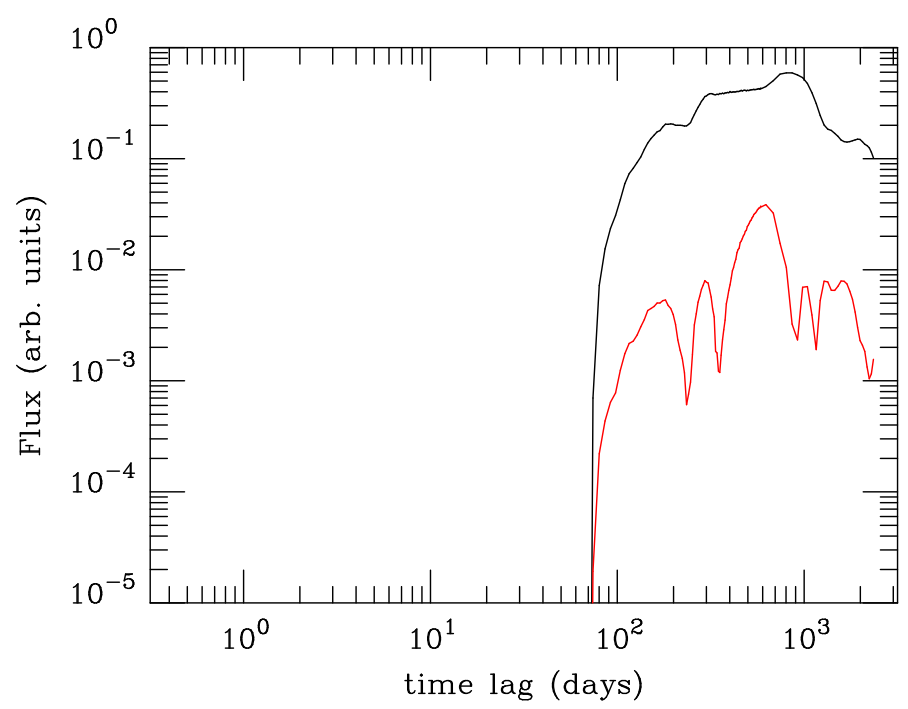

Fig. 4. Same as Fig. 3 in the clumpy dust torus case.

Much longer delays are expected in the case of a clumpy medium because photons can penetrate much deeper than in the uniform case; the depth they can reach depends on the filling factor and on the optical depth of individual blobs. It typically varies as the filling factor to the power $-2 / 3$. However, significant deviations are expected because the cloud radius $(0.2-0.6 \mathrm{pc})$ is larger than the inner radius of the disk. $\Psi(\tau)$ is shown in Fig. 4, and the peak of $\tau \Psi(\tau)$ occurs at $\tau=650$ days, almost an order of magnitude larger than in the uniform case. This compares well with $\tau_{\mathrm{m}}=706 \mathrm{~d}$, and is smaller than the mean $\langle\tau\rangle=1240 \mathrm{~d}$. $\Psi$ also shows significant fluctuations that reveal individual blobs.

\subsubsection{Polar wind}

Figure 5 shows the transfer function for the polar wind case. The delays are long because the scattering region extends over large distances from the central source. In contrast with the previous cases, $\Psi$ is monotonic; the first plateau, for lags up to a few thousand days, corresponds to the fraction of the wind flowing toward the observer; the second one, for lags of 10000 


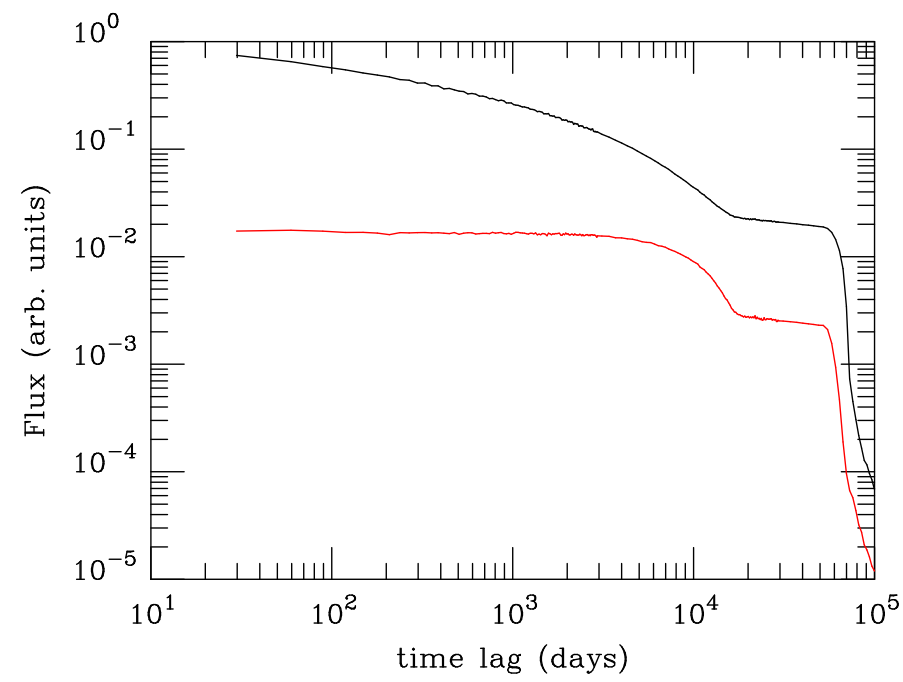

Fig. 5. Same as Fig. 3 in the polar wind case.

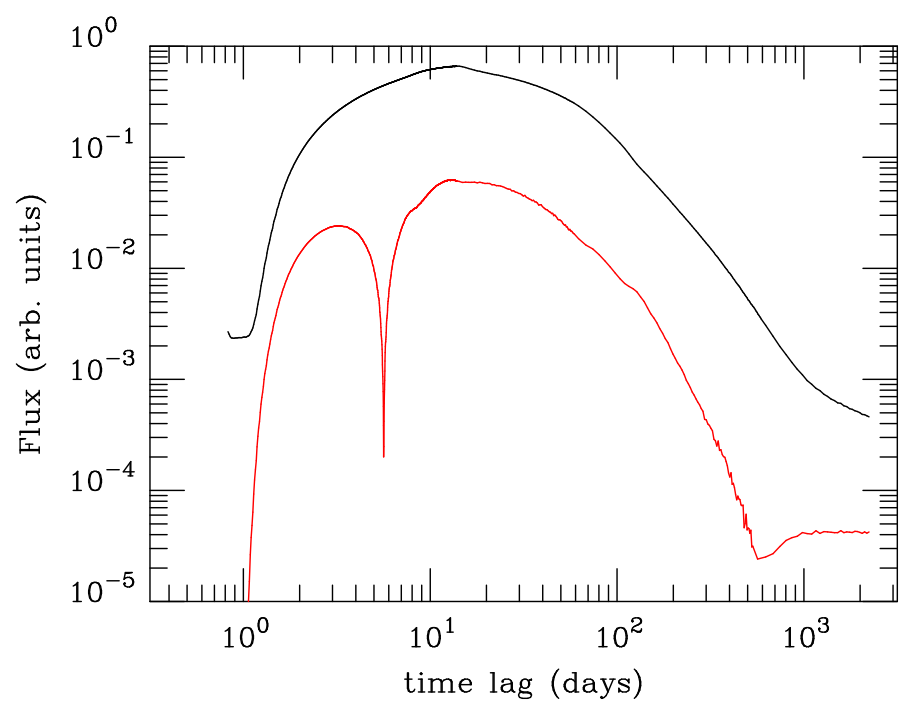

Fig. 6. Same as Fig. 3 when all components of the AGN (uniform dust torus, polar wind and equatorial ring) are included.

to about 30000 days, is produced by the fraction of the wind flowing away from the observer. The tail for $\tau$ in the range $7 \times 10^{4}-10^{5}$ days is produced by photons that have scattered more than once. There are two peaks of $\tau \Psi(\tau)$; the first is obtained for $\tau=37 \mathrm{yr}$, and the second at $\tau=129 \mathrm{yr}$, with a corresponding value of $\tau \Psi(\tau)$ higher than the first peak only by a factor 1.15 . The median lag is $33 \mathrm{yr}$, while the mean is $49 \mathrm{yr}$, clearly corresponding to the first of the two peaks.

\subsubsection{Full model}

Finally, Fig. 6 shows $\Psi(\tau)$ when all components of the AGN are included: torus, polar wind, and equatorial ring. Here, the torus is assumed to be uniform. The peak in $\tau \Psi(\tau)$ is obtained for $\tau=39$ days; in the case of a clumpy torus, this maximum is reduced to 33 days because in the clumpy torus case, $\Psi(\tau)$ is nonvanishingly small for a much broader range of $\tau$, which tends to dilute its effect on the global curve, and also because the clumpy torus introduces delays much longer than the BLR, and has no influence on $\Psi$ for values of $\tau$ of about 25 days.

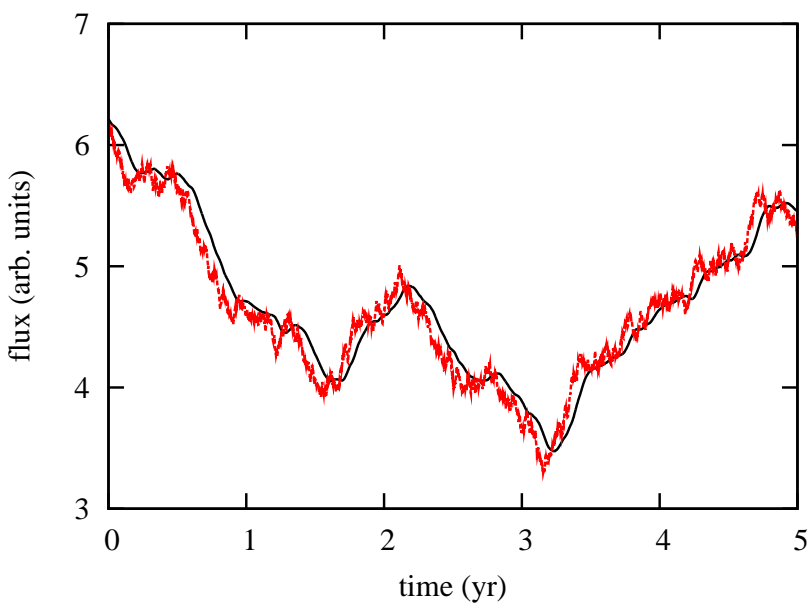

Fig. 7. Total (red) and polarized (black) light curves obtained convolving the source term with the transfer function for a geometry including a dust torus, a polar wind, and an equatorial scattering ring with an optical depth of one. The polarized flux has been rescaled by about one order of magnitude so that it can easily be compared to the total flux.

\subsection{Cross-correlation analysis}

Figure 7 shows the total and polarized fluxes calculated using Eq. (1) for a geometry including a dust torus, a polar wind, and an equatorial scattering ring; the parameters are given in Table 1. The effect of the scattering ring on the polarized radiation is clearly visible; it both smooths the short-term fluctuations and introduces a delay that is visible to the eye, on timescales as short as a few days.

We then performed a cross-correlation analysis of the total and polarized flux after removing the long-term trend by subtracting the linear component of the light curve, defined as $L(0)+(L(T)-L(0)) t / T$, where $T$ is the observation duration. The cross correlation has a maximum for a positive time lag, but the position of the maximum varies somewhat for different source light curves. We thus generated for each configuration 100 different light curves, which enabled us to estimate the mean delay and its standard deviation. Subtracting the long-term behavior of the light curve is essential to reduce the standard deviation of the measured time lag; the accuracy of the determination of this delay is improved by a factor larger than 10 . The reason is that for the timescales we consider here, the mean and standard deviation are difficult to estimate because of the importance of the low-frequency component in the light curves (see, e.g., Vio \& Wamsteker 2001, for a discussion of the effect of the limits of the cross-correlation analysis in the case of short time-series). Increasing the integration time does not solve the problem because of the $v^{-2}$ frequency dependence of the signal in the case of a random walk.

We also stress that our modeled light curves do not include statistical and instrumental noise, nor gaps in data collection. These introduce observational errors and possibly biases in the measured lags. For lags of about a few days to a few weeks, the fact that sources are observable only at night during a fraction of a year is no limitation, but the availability of telescope time might be a problem. In any case, real observations will be analyzed using more sophisticated methods, such as the discrete correlation function (DCF) or $z$-transformed DCF (ZDCF, Alexander 2013). These methods also allow estimating the observational error on the lag. We used here a simplified method because we are not limited by observational constraints, 
Table 2. Time lag (in days) between the polarized and total flux for different configurations.

\begin{tabular}{lccc}
\hline \hline Component & $V(5500 \AA)$ & $B(4500 \AA)$ & UV $(2500 \AA)$ \\
\hline BLR & $26.2 \pm 0.9$ & $26.4 \pm 0.9$ & $26.2 \pm 0.8$ \\
Uniform dust torus & $90.7 \pm 2.4$ & $84.0 \pm 2.0$ & $68.5 \pm 1.8$ \\
Clumpy dust torus & $686 \pm 24$ & $695 \pm 13$ & $678 \pm 17$ \\
Polar wind & $11500 \pm 850$ & $11500 \pm 730$ & $11700 \pm 810$ \\
Uniform dust torus + BLR & $29.1 \pm 1.0$ & $29.5 \pm 1.0$ & $28.3 \pm 1.2$ \\
Uniform dust torus + polar wind & $96.0 \pm 3.9$ & $89.7 \pm 3.0$ & $73.2 \pm 2.6$ \\
Uniform dust torus + polar wind + BLR & $29.6 \pm 1.2$ & $29.0 \pm 1.1$ & $28.5 \pm 0.8$ \\
Uniform flared disk + BLR & $28.5 \pm 1.0$ & $28.3 \pm 1.3$ & $27.9 \pm 0.7$ \\
Uniform flared disk + polar wind & $97.5 \pm 4.9$ & $92.8 \pm 5.0$ & $65.8 \pm 2.5$ \\
Uniform flared disk + polar wind + BLR & $29.1 \pm 1.1$ & $28.5 \pm 1.2$ & $28.2 \pm 0.8$ \\
Clumpy dust torus + BLR & $26.6 \pm 0.7$ & $25.9 \pm 0.8$ & $26.3 \pm 0.9$ \\
Clumpy dust torus + polar wind & $8260 \pm 1200$ & $8600 \pm 1200$ & $8750 \pm 1300$ \\
Clumpy dust torus + polar wind + BLR & $27.1 \pm 0.9$ & $27.3 \pm 0.9$ & $27.6 \pm 0.8$ \\
\hline
\end{tabular}

and we note that by construction, the DCF or the ZCDF method will provide the same results as ours on the time lag for a continuous long observational time.

\section{Results}

Table 2 gives the time lag in days between the polarized and total flux for various configurations and for three observation wavelengths: $5500 \AA$, corresponding to the $V$ band, $4500 \AA$ for the $B$ band, and $2500 \AA$ in the ultraviolet. The lags we find are not very different from those obtained when searching for the maximum of $\tau \Psi(\tau)$, as discussed in the previous section, with the exception of the polar wind case. In the latter case, the cross-correlation process picks up the first peak in $\tau \Psi(\tau)$. As expected, the delay obtained when the BLR and the polar wind alone are considered does not depend on the observation wavelength because Thomson scattering is a wavelength-independent process. The uniform dusty environments (flared disk or torus) generate wavelengthdependent delays, as expected, but time lags in clumpy environments do not depend on wavelength. This occurs because the depth to which photons can penetrate depends on the distribution of clumps and is thus wavelength independent, while the absorption process itself still depends on wavelength, but because the clumps are optically thick (the optical depth of each cloud is 50), this occurs only at the surface of the clumps.

Our main result is that regardless of the configuration, the time lag is determined by the presence or absence of the BLR. When present, it essentially sets the lag to about 25 days; this lag is independent of the observation wavelength. It corresponds approximately to the outer radius of the BLR, thereby validating the method of Gaskell et al. (2012) to constrain the physical characteristics of the BLR. As mentioned in the introduction, we do not intend to reproduce the observed delay in one particular object, but rather to test the method.

It is interesting to compare these results to the average time lag we estimated in Paper I and defined in Eq. (5). This average delay is significantly longer than the time lag found when the peak of the cross-correlation function was calculated. In the case of a uniform dusty torus plus polar winds and BLR, this average time is about 1.7 years, much longer than the 25 days found here.

We can also estimate the time lag between the polarized and total flux for different optical depths of the BLR. The results are given in Table 3. As expected, the higher the optical depth, the shorter the lag because for high optical depths, photons
Table 3. Time lag (in days) between the polarized and total flux for different configurations.

\begin{tabular}{lc}
\hline \hline$\tau_{\text {BLR }}$ & Lag \\
\hline 1 & $26.2 \pm 0.9$ \\
3 & $16.2 \pm 0.5$ \\
10 & $7.3 \pm 0.3$ \\
\hline
\end{tabular}

cannot penetrate deeply into the equatorial ring and are scattered in regions closer to the inner edge of the ring. For reasonable values of the optical depths of the BLR (of about one), the lag is expected to be about 25 days.

The accuracy to which the time lag can be measured depends indeed on the observing time. The values presented in Table 2 are calculated using very long baselines, much longer than what would be realistic: we used a duration of 80 years for the uniform or clumpy torus plus polar wind and equatorial ring. For these long observing times, the uncertainty on our results partly arises from the discretization noise because our time resolution is $0.6 \mathrm{~d}$. For observations lasting for five years, this lag is still measurable with an accuracy of 7 days, and it remains detectable for observing times as short as one year. We stress that the possibility of determining the time lag with a reasonable degree of confidence strongly depends on the statistics of the light fluctuations, which are strongly source dependent. For one given source, the ability of determining a time lag also depends on the specific realization of the light-curve noise, as can be anticipated by a closer look at Fig. 7. The most favorable situation occurs when a peak (or a minimum) whose width is slightly larger than the expected time lag is detected during one observation, in which case the total observing time need not be longer than a few months. We finally note that the noise properties we chose here (random walk generating a $v^{-2}$ power spectrum) are not favorable at all because of the low frequencies, making it difficult (formally impossible) to determine the mean and standard deviation of the light curve.

We verified that the time lags we obtained do not depend sensitively on the assumption made to describe the time variability of the central source. A value of $\beta$ in Eq. (3) ten times higher or lower than our reference value of 0.07 leads to delays for the clumpy dusty torus (CDT) + polar wind (PW) + BLR model that are within our uncertainties. Similarly, we considered a damped random walk model with a characteristic damping time of 20, 200, and 2000 days. We obtained for the CDT+PW+BLR 
models lags of $22.3 \pm 0.3,23.0 \pm 0.3$, and $24.2 \pm 1.8$ days, respectively, which agrees well with the value of 26.2 days obtained in the pure random walk case. This is expected because we interpret the time-lag as the maximum of $\tau \Psi(\tau)$.

\section{Conclusion}

We have shown that determining the delay between the polarized and total flux using cross-correlation techniques allows constraining the size of the BLR, as first suggested by Gaskell et al. (2012). Matter located at larger distance, as in the dust torus or in polar winds, for instance, does not contribute to the time lag as determined using this technique, although it does increase the average propagation time of a photon. The reason is that this external matter does not significantly modify the peak of the transfer function, but introduces wings that can be significant enough to change the mean travel time by a large factor. This difference between the time lag determined by cross-correlation techniques and the average propagation time delay also arises because short-term fluctuations in the source light curve may prevent one from finding correlations on very long timescales. The only case where the outer environment may affect the measured time lag is when the time lag generated by these regions is commensurable with the lag due to the BLR; in our case when the dust torus is not clumpy. This effect is moderate in any case.

We also showed that this time lag is measurable as long as the observing time span covered by observations is longer than a few years, and possibly shorter, depending on the statistical properties of the light fluctuations of a given source or on the actual realisation of the noise.

We note, however, that the time lag between the polarized and unpolarized radiation does not univocally determine the BLR size because parameters such as in particular the AGN viewing angle and the BLR optical depth must be known a priori. These are reasonably constrained: the viewing angle must not be large for a type 1 AGN, and the BLR optical depth cannot be significantly higher than unity. Their uncertainty can be significant, however (see, e.g., Marin 2016, for a discussion of the determination of the inclination angle). We also considered a homogeneous scattering ring; clumps are certainly present in the BLR (Gaskell 2009), but because the BLR optical depth cannot be high, the presence of clumps is not as dramatic as it is in the case of a dust torus, for instance.

Better constraints might be obtained by considering polarized lines, as discussed in Popovic et al. (2018) and Savić et al. (2018), for example, because the width of the line directly measures the radial velocity of the material and is thus connected to the position of the emitting material in the BLR. We leave this for a future work.

As a final remark, it is worth mentioning that this method can be used for low-luminosity sources, such as the Galactic center. The immediate vicinity of Sgr A* contains several gaseous structures (Ciurlo et al. 2020; Peißker et al. 2020) whose nature is still uncertain; they are unresolved, and at least for the so-called $\mathrm{G}$ objects, they show emission properties of gas clouds, but their dynamical properties are typical of stellar objects. One of them, G2, is intrinsically linearly polarized (Shahzamanian et al. 2016) in the infrared ( $K_{s}$ band), with a polarized fraction higher than $20 \%$, and a varying polarization angle as it approaches the position of Sgr A*. If the infrared luminosity of these clouds at least for a sizeable fraction is caused by the reprocessing of $\operatorname{Sgr} \mathrm{A}^{*}$ luminosity, we would expect a delayed response of the polarized radiation of these sources with respect to Sgr A*.

Acknowledgements. We are grateful to D. Savic and M. Gaskell for helpful discussions. We are grateful to the referee for helpful comments and suggestions, in particular concerning the applicability of our method to the Galactic centre. This research has been supported by the French Programme National des Hautes Energies (PNHE). FM is grateful for CNES funding under the post-doctoral grant "Probing the geometry and physics of active galactic nuclei with ultraviolet and $\mathrm{X}$-ray polarized radiative transfer". APRL acknowledge support from the CONICYT BECAS Chile grant no. 72150573.

\section{References}

Alexander, T. 2013, ArXiv e-prints [arXiv:1302 . 1508]

Antonucci, R. 1993, ARA\&A, 31, 473

Antonucci, R. R. J. 1984, ApJ, 278, 499

Antonucci, R. R. J., \& Miller, J. S. 1985, ApJ, 297, 621

Aranzana, E., Körding, E., Uttley, P., Scaringi, S., \& Bloemen, S. 2018, MNRAS, 476, 2501

Bentz, M. C., Denney, K. D., Grier, C. J., et al. 2013, ApJ, 767, 149

Blandford, R. D., \& McKee, C. F. 1982, ApJ, 255, 419

Chandrasekhar, S. 1960, Radiative Transfer (New York, USA: Dover Publications)

Ciurlo, A., Campbell, R. D., Morris, M. R., et al. 2020, Nature, 577, 337

Czerny, B., \& Hryniewicz, K. 2011, A\&A, 525, L8

Czerny, B., Li, Y.-R., Hryniewicz, K., et al. 2017, ApJ, 846, 154

Czerny, B., Olejak, A., Rałowski, M., et al. 2019, ApJ, 880, 46

Event Horizon Telescope Collaboration (Akiyama, K., et al.) 2019, ApJ, 875, L1 García-Burillo, S., Combes, F., Ramos Almeida, C., et al. 2016, ApJ, 823, L12

Gaskell, C. M. 2009, New Astron. Rev., 53, 140

Gaskell, C. M., Goosmann, R. W., Merkulova, N. I., Shakhovskoy, N. M., \& Shoji, M. 2012, ApJ, 749, 148

Goosmann, R. W., \& Gaskell, C. M. 2007, A\&A, 465, 129

Gravity Collaboration (Sturm, E., et al.) 2018a, Nature, 563, 657

Gravity Collaboration (Abuter, R., et al. 2018b, A\&A, 618, L10

Gravity Collaboration (Dexter, J., et al.) 2020, A\&A, 635, A92

Jaffe, W., Meisenheimer, K., Röttgering, H., Leinert, C., \& Richichi, A. 2004, in

The Interplay Among Black Holes, Stars and ISM in Galactic Nuclei, eds. T.

Storchi-Bergmann, L. C. Ho, \& H. R. Schmitt, IAU Symp., 222, 37

Kelly, B. C., Bechtold, J., \& Siemiginowska, A. 2009, ApJ, 698, 895

Kelly, B. C., Becker, A. C., Sobolewska, M., Siemiginowska, A., \& Uttley, P. 2014, ApJ, 788, 33

Lira, P., Kaspi, S., Netzer, H., et al. 2018, ApJ, 865, 56

MacLeod, C. L., Ivezić, Ž., Kochanek, C. S., et al. 2010, ApJ, 721, 1014

Marin, F. 2016, MNRAS, 460, 3679

Marin, F. 2018, A\&A, 615, A171

Marin, F., \& Goosmann, R. W. 2013, MNRAS, 436, 2522

Marin, F., Goosmann, R. W., Gaskell, C. M., Porquet, D., \& Dovčiak, M. 2012, A\&A, 548, A121

Marin, F., Goosmann, R. W., \& Gaskell, C. M. 2015, A\&A, 577, A66

Miller, J. S., \& Antonucci, R. R. J. 1983, ApJ, 271, L7

Peißker, F., Hosseini, S. E., Zajaček, M., et al. 2020, A\&A, 634, A35

Peterson, B. M. 1993, PASP, 105, 247

Popovic, L. C., Afanasiev, V. L., \& Savic, D. 2018, ArXiv e-prints [arXiv:1807.00177]

Raban, D., Jaffe, W., Röttgering, H., Meisenheimer, K., \& Tristram, K. R. W. 2009, MNRAS, 394, 1325

Rojas Lobos, P. A., Goosmann, R. W., Marin, F., \& Savić, D. 2018, A\&A, 611, A39 (Paper I)

Savić, D., Goosmann, R., Popović, L. Č., Marin, F., \& Afanasiev, V. L. 2018, A\&A, 614, A120

Shahzamanian, B., Eckart, A., Zajaček, M., et al. 2016, A\&A, 593, A131

Shoji, M., Gaskell, C. M., \& Goosmann, R. W. 2005, in American Astronomical Society Meeting Abstracts, BAAS, 1420, 37

Smith, J. E., Robinson, A., Alexander, D. M., et al. 2004, MNRAS, 350, 140

Timmer, J., \& Koenig, M. 1995, A\&A, 300, 707

Tombesi, F., Cappi, M., Reeves, J. N., et al. 2013, MNRAS, 430, 1102

Vio, R., \& Wamsteker, W. 2001, PASP, 113, 86

Zu, Y., Kochanek, C. S., \& Peterson, B. M. 2011, ApJ, 735, 80

Zu, Y., Kochanek, C. S., Kozłowski, S., \& Udalski, A. 2013, ApJ, 765, 106 